\title{
A RESULT BY SILVERMAN TRANSFERRED ON MULTIVALENT FUNCTIONS
}

\author{
E. Karamazova And N. Tuneski
}

ABSTRACT. In 1999 Siverman introduced a class of univalent functions defined over the quotient of the analytical representations of convexity and starlikeness. This class was widely studied afterwards. In this paper, in a similar manner, we define a class of multivalent functions and obtain results, dual to the ones for univalent functions.

2010 Mathematics Subject Classification: 30C45, 30C55.

Keywords: analytic function; multivalent function; class defined by Silverman.

\section{INTRODUCTION}

For $n \in \mathbb{N}$ and $a \in \mathbb{C}$ let

$$
\mathcal{H}[a, n]=\left\{f \in \mathcal{H}(\mathbb{D}): f(z)=a+a_{n} z^{n}+a_{n+1} z^{n+1}+\cdots\right\},
$$

where $\mathcal{H}(\mathbb{D})$ is the class of all functions that are analytic in the open unit disk $\mathbb{D}=\{z \in \mathbb{C}:|z|<1\}$. Also, let for a positive integer $p, \mathcal{A}_{p}$ be the subclass of $H(\mathbb{D})$ consisting of functions of the form $f(z)=z^{p}+a_{p+1} z^{p+1}+\cdots$ and $\mathcal{A} \equiv \mathcal{A}_{1}$, so that $\mathcal{A}$ is the class of functions $f$ which are analytic in $\mathbb{D}$ with normalization $f(0)=0$ and $f^{\prime}(0)=1$. More details in $[2,3,9]$.

A function $f$ is said to be multivalent or $p$-valent in $\mathbb{D}$ if it takes no value more than $p$ times in $\mathbb{D}$ and there is some $\omega_{0}$ such that $f(z)=\omega_{0}$ has exactly $p$ solutions in $\mathbb{D}$, when roots are counted in accordance with their multiplicities.

The classes of $p$-valently starlike functions of order $\alpha$ and $p$-valently convex functions of order $\alpha(p=1,2, \ldots$ and $0 \leq \alpha<p)$ are, respectively, defined by

$$
\mathcal{S}_{p}^{*}(\alpha)=\left\{f \in \mathcal{A}_{p}: \operatorname{Re}\left[\frac{z f^{\prime}(z)}{f(z)}\right]>\alpha, z \in \mathbb{D}\right\}
$$

and

$$
\mathcal{K}_{p}(\alpha)=\left\{f \in \mathcal{A}_{p}: \operatorname{Re}\left[1+\frac{z f^{\prime \prime}(z)}{f^{\prime}(z)}\right]>\alpha, z \in \mathbb{D}\right\}
$$


E. Karamazova and N. Tuneski - A result by Silver. transf. on multival. func.

For $p=1$, we have respectively, the usual classes of starlike and convex functions of order $\alpha, \mathcal{S}^{*}(\alpha)$ and $\mathcal{K}(\alpha)$; and for $p=2,3, \ldots$, we have the classes of multivalent starlike and convex functions of order $\alpha$. In the case $\alpha=0$, we will use the notations $\mathcal{S}_{p}^{*}$ and $\mathcal{K}_{p}$; and in the case $\alpha=0$ and $p=1$, the notations $\mathcal{S}^{*}$ and $\mathcal{K}$.

\section{PReliminaries}

Silverman in his work [7] investigated the properties of a class of functions defined in terms of the quotient of the analytical representations of convexity and starlikeness, $\frac{1+z f^{\prime \prime}(z) / f^{\prime}(z)}{z f^{\prime}(z) / f(z)}$. Precisely, he considered the following classes

$$
\mathcal{G}_{b}=\left\{f \in \mathcal{A}:\left|\frac{1+z f^{\prime \prime}(z) / f^{\prime}(z)}{z f^{\prime}(z) / f(z)}-1\right|<b, z \in \mathbb{D}\right\}
$$

$0<b \leq 1$. This class was widely studied ever since (see $[8,6,10,11]$ )

In this paper we study an analogue expression for multivalent functions,

$$
\frac{1+z f^{(p+1)}(z) / f^{(p)}(z)}{z f^{(p)}(z) / f^{(p-1)}(z)}
$$

and find sufficient conditions so that the following implication holds:

$$
\left|\frac{1+z f^{(p+1)}(z) / f^{(p)}(z)}{z f^{(p)}(z) / f^{(p-1)}(z)}-1\right|<\lambda \Rightarrow\left|\frac{z f^{\prime}(z)}{f(z)}-p\right|<\mu
$$

(both inequalities are on the whole unit disk).

For that purpose we use a method from the theory of differential subordinations to get our main result. More on this theory can be found in [3] and [1].

First we introduce the concept of subordination. Let $f(z), g(z) \in \mathcal{A}$. We say that $f(z)$ is subordinate to $g(z)$, and write $f(z) \prec g(z)$, if there exists a function $\omega(z)$, analytic in the unit disc $\mathbb{D}$, such that $\omega(0)=0,|\omega(z)|<1$ and $f(z)=g(\omega(z))$ for all $z \in \mathbb{D}$. If $g(z)$ is univalent in $\mathbb{D}$ then $f(z) \prec g(z)$ if and only if $f(0)=g(0)$ and $f(\mathbb{D}) \subseteq g(\mathbb{D})$.

The general theory of differential subordinations, as well as the theory of firstorder differential subordinations, was introduced by Miller and Mocanu in [4] and [5]. In fact, if $\phi: C^{2} \rightarrow \mathbb{C}, \mathbb{C}$ complex plane, is analytic in a domain $D$, if $h(z)$ is univalent in $\mathbb{D}$, and if $p(z)$ is analytic in $\mathbb{D}$ with $\left(p(z), z p^{\prime}(z)\right) \in D$ when $z \in \mathbb{D}$, then $p(z)$ is said to satisfy a first-order differential subordination if

$$
\phi\left(p(z), z p^{\prime}(z)\right) \prec h(z) .
$$


E. Karamazova and N. Tuneski - A result by Silver. transf. on multival. func.

A univalent function $q(z)$ is called a dominant of the differential subordination (2) if $p(z) \prec q(z)$ for all $p(z)$ satisfying (2). If $\widetilde{q}(z)$ is a dominant of $(2)$ and $\widetilde{q}(z) \prec q(z)$ for all dominants of $(2)$, then we say that $\widetilde{q}(z)$ is the best dominant of the differential subordination (2).

For proving implications in this paper we will use the following lemma from the theory of differential subordinations. She give efficient tool for obtaining sufficient conditions when certain differential inequality holds.

Lemma 1 (Theorem $2.3 \mathrm{~h}(\mathrm{i}), \mathrm{p} .34,[3]$ ). Let $\Omega \subset \mathbb{C}$ and suppose that the function $\psi: \mathbb{C}^{2} \times \mathbb{D} \rightarrow \mathbb{C}$ satisfies $\psi\left(M e^{i \theta}, K e^{i \theta} ; z\right) \notin \Omega$ for all $K \geq M n, \theta \in \mathbb{R}$ and $z \in \mathbb{D}$. If $p \in \mathcal{H}[a, n]$ and $\psi\left(p(z), z p^{\prime}(z) ; z\right) \in \Omega$ for all $z \in \mathbb{D}$, then $|p(z)|<M, z \in \mathbb{D}$.

\section{MAIN RESUlts}

In the beginning we will study implication:

$$
\left|\frac{1+z f^{(p+1)}(z) / f^{(p)}(z)}{z f^{(p)}(z) / f^{(p-1)}(z)}-1\right|<\lambda \quad \Rightarrow \quad \frac{z f^{(p)}(z)}{f^{(p-1)}(z)}-1 \mid<\lambda_{1}
$$

(both inequalities are on the whole unit disk), that will later lead to the implication (1).

Theorem 2. Let $p$ be a positive integer, $p \geq 2$ and $\lambda_{1}>0$. Also, let $f \in \mathcal{A}_{p}$. If $\lambda \leq \lambda\left(\lambda_{1}\right) \equiv \frac{\lambda_{1}}{\left(\lambda_{1}+1\right)^{2}}$, then implication (3) holds.

Proof. In the view of Lemma 1 , let put $M=\lambda_{1}$ and let define function $p(z)=$ $\frac{z f^{(p)}(z)}{f^{(p-1)}(z)}-1$. Further, for the function $\psi(r, s ; z)=\frac{s}{(r+1)^{2}}$, such that $p(z) \in \mathcal{H}[0,1]$ $(n=1$ in the Lemma 1$)$ we have

$$
\psi\left(p(z), z p^{\prime}(z) ; z\right)=\left|\frac{z p^{\prime}(z)}{(p(z)+1)^{2}}\right|=\left|\frac{1+z f^{(p+1)}(z) / f^{(p)}(z)}{z f^{(p)}(z) / f^{(p-1)}(z)}-1\right|<\lambda\left(\lambda_{1}\right)
$$

for all $z \in \mathbb{D}$ and

$$
\begin{gathered}
\psi\left(M e^{i \theta}, K e^{i \theta} ; z\right)=\left|\frac{K e^{i \theta}}{\left(M e^{i \theta}+1\right)^{2}}\right|=\frac{K\left|e^{i \theta}\right|}{\left|M e^{i \theta}+1\right|^{2}}= \\
=\frac{K}{\left|M e^{i \theta}+1\right|^{2}} \geq \frac{M}{\left|M e^{i \theta}+1\right|^{2}} \geq \frac{M}{M^{2}+2 M+1}= \\
=\frac{M}{(M+1)^{2}}=\frac{\lambda_{1}}{\left(\lambda_{1}+1\right)^{2}} \geq \lambda
\end{gathered}
$$

for all $z \in \mathbb{D}$. So, $|p(z)|<M$ for all $z \in \mathbb{D}$. 
E. Karamazova and N. Tuneski - A result by Silver. transf. on multival. func.

In a similar way we prove the following result.

Theorem 3. Let $p$ and $k$ are integers such that $p \geq 2$ and $0 \leq k \leq p-2$. Also let $f \in \mathcal{A}_{p}, \lambda_{k+1}>0$ and

$$
\lambda_{k} \leq \lambda_{k}\left(\lambda_{k+1}\right) \equiv \frac{\lambda_{k+1}}{\lambda_{k+1}+k+2}+\lambda_{k+1} .
$$

If

$$
\left|\frac{z f^{(p-k)}(z)}{f^{(p-k-1)}(z)}-(k+1)\right|<\lambda_{k}\left(\lambda_{k+1}\right) \quad(z \in \mathbb{D}),
$$

then

$$
\left|\frac{z f^{(p-k-1)}(z)}{f^{(p-k-2)}(z)}-(k+2)\right|<\lambda_{k+1} \quad(z \in \mathbb{D}) .
$$

Proof. Let put $M=\lambda_{k+1}$ and let define functions

$$
p(z)=\frac{z f^{(p-k-1)}(z)}{f^{(p-k-2)}(z)}-k-2
$$

and

$$
\psi(r, s ; z)=\frac{s}{r+k+2}+r,
$$

such that $p(z) \in \mathcal{H}[0,1](n=1$ in the Lemma 1$)$ and

$$
\begin{aligned}
\psi\left(p(z), z p^{\prime}(z) ; z\right) & =\left|\frac{z p^{\prime}(z)}{p(z)+k+2}+p(z)\right| \\
& =\left|\frac{z f^{(p-k)}(z)}{f^{(p-k-1)}(z)}-k-1-p(z)+p(z)\right| \\
& =\left|\frac{z f^{(p-k)}(z)}{f^{(p-k-1)}(z)}-k-1\right|<\lambda_{k}\left(\lambda_{k+1}\right)
\end{aligned}
$$

for all $z \in \mathbb{D}$. In order to prove the theorem it is enough to show that $\psi\left(M e^{i \theta}, K e^{i \theta} ; z\right) \geq$ $\lambda_{k}\left(\lambda_{k+1}\right)$. Indeed, since $k+2 \geq M$ we have

$$
\begin{aligned}
\psi\left(M e^{i \theta}, K e^{i \theta} ; z\right) & =\left|\frac{K e^{i \theta}}{M e^{i \theta}+k+2}+M e^{i \theta}\right|=\left|\frac{K}{M e^{i \theta}+k+2}+M\right| \\
& \geq \frac{K}{M+k+2}+M \geq \frac{M}{M+k+2}+M \\
& =\frac{\lambda_{k+1}}{\lambda_{k+1}+k+2}+\lambda_{k+1}=\lambda_{k}\left(\lambda_{k+1}\right)
\end{aligned}
$$

for all $z \in \mathbb{D}$. 
E. Karamazova and N. Tuneski - A result by Silver. transf. on multival. func.

Since the functions $\lambda\left(\lambda_{1}\right)=\frac{\lambda_{1}}{\left(\lambda_{1}+1\right)^{2}}$ and $\lambda_{k}\left(\lambda_{k+1}\right)=\frac{\lambda_{k+1}}{\lambda_{k+1}+k+2}+\lambda_{k+1}$ are strictly monotone on the interval $(0,1)$ and $(0,+\infty)$, respectively, we realize that there exist their inverse $\lambda_{1}(\lambda)=\frac{1-2 \lambda+\sqrt{1-4 \lambda}}{2 \lambda}$ and

$$
\lambda_{k+1}\left(\lambda_{k}\right)=\frac{1}{2}\left(\lambda_{k}-k-3+\sqrt{\left(\lambda_{k}+k+3\right)^{2}-4 \lambda_{k}}\right) .
$$

So, Theorem 2 and Theorem 3 can be rewritten in the following, equivalent form.

Theorem 4. Let $p$ be a positive integer, $p \geq 2$ and $0<\lambda \leq 1 / 4$. Also, let $f \in \mathcal{A}_{p}$. If

$$
\left|\frac{1+z f^{(p+1)}(z) / f^{(p)}(z)}{z f^{(p)}(z) / f^{(p-1)}(z)}-1\right|<\lambda \quad(z \in \mathbb{D}),
$$

then

$$
\left|\frac{z f^{(p)}(z)}{f^{(p-1)}(z)}-1\right|<\frac{1-2 \lambda+\sqrt{1-4 \lambda}}{2 \lambda} \quad(z \in \mathbb{D}) .
$$

Theorem 5. Let $p$ and $k$ are integers such that $p \geq 2$ and $0 \leq k \leq p-2$. Also let $f \in \mathcal{A}_{p}$ and $0<\lambda_{k}<1$. If

$$
\left|\frac{z f^{(p-k)}(z)}{f^{(p-k-1)}(z)}-(k+1)\right|<\lambda_{k} \quad(z \in \mathbb{D}),
$$

then

$$
\begin{aligned}
\left|\frac{z f^{(p-k-1)}(z)}{f^{(p-k-2)}(z)}-(k+2)\right| & <\lambda_{k+1} \\
& \equiv \frac{1}{2}\left[\lambda_{k}-k-3+\sqrt{\left(\lambda_{k}+k+3\right)^{2}-4 \lambda_{k}}\right] \quad(z \in \mathbb{D}) .
\end{aligned}
$$

We reach the final goal of this paper by combining the two previous theorems. Theorem 5 can be applied recursively because $\frac{1}{2}\left[\lambda_{k}-k-3+\sqrt{\left(\lambda_{k}+k+3\right)^{2}-4 \lambda_{k}}\right]$ is in the interval $(0,1)$ when $\lambda_{k} \in(0,1)$. 
E. Karamazova and N. Tuneski - A result by Silver. transf. on multival. func.

Theorem 6. Let $p$ be a positive integer, $p \geq 2$ and $0<\lambda \leq 1 / 4$. Also, let $\lambda_{1}=$ $\frac{1-2 \lambda+\sqrt{1-4 \lambda}}{2 \lambda}$ and for $k=1,2, \ldots$,

$$
\lambda_{k+1}=\frac{1}{2}\left[\lambda_{k}-k-3+\sqrt{\left(\lambda_{k}+k+3\right)^{2}-4 \lambda_{k}}\right] .
$$

If $f \in \mathcal{A}_{p}$ and

$$
\left|\frac{1+z f^{(p+1)}(z) / f^{(p)}(z)}{z f^{(p)}(z) / f^{(p-1)}(z)}-1\right|<\lambda \quad(z \in \mathbb{D}),
$$

then

$$
\left|\frac{z f^{\prime}(z)}{f(z)}-p\right|<\lambda_{p-1} \quad(z \in \mathbb{D})
$$

For $p=2$ and $\lambda=1 / 4$ we have $\lambda_{1}=1$ and receive the following corollary.

Corollary 7. If $f \in \mathcal{A}_{2}$ and

$$
\left|\frac{1+z f^{\prime \prime \prime}(z) / f^{\prime \prime}(z)}{z f^{\prime \prime}(z) / f^{\prime}(z)}-1\right|<\frac{1}{4} \quad(z \in \mathbb{D}),
$$

then

$$
\left|\frac{z f^{\prime}(z)}{f(z)}-2\right|<1 \quad(z \in \mathbb{D})
$$

\section{REFERENCES}

[1] T. Bulboaca, Differential subordinations and superordinations. New results, House of Science Book Publ., Cluj-Napoca (2005).

[2] P.L. Duren, Univalent functions, Springer-Verlag (1983).

[3] S.S. Miller, P.T. Mocanu, Differential subordinations: Theory and Applications, Marcel Dekker, New York-Basel (2000).

[4] S.S. Miller, P.T. Mocanu, Differential subordinations and univalent functions, Michigan Math. J. 28, 2 (1981), 157-172.

[5] S.S. Miller, P.T. Mocanu, On some classes of first-order differential subordinations, Michigan Math. J. 32 (1985), 185-195.

[6] M. Obradović, N. Tuneski, On the starlike criteria defined by Silverman, Zeszyty Nauk. Politech. Rzeszowskiej Mat. 181, 24 (2000), 59-64.

[7] H. Silverman, Convex and starlike criteria, Int. J. Math. Math. Sci. 22, 1 (1999), 75-79. 
E. Karamazova and N. Tuneski - A result by Silver. transf. on multival. func.

[8] V. Singh, N. Tuneski, On a Criteria for Starlikeness and Convexity of Analytic Functions, Acta Mathematica Scientia 24 No. B4 (2004), 597-602.

[9] H.M. Srivastava, S. Owa, Current topics in analytic function theory, World Sci. Publ., River Edge, NJ (1992).

[10] N. Tuneski, On the quotient of the representations of convexity and starlikeness, Math. Nachr. 248/249 (2003), 200-203.

[11] N. Tuneski, On a criteria for starlikeness of analytic functions, Integral Transforms Spec. Funct. 14, 3 (2003), 263-270.

Elena Karamazova

Faculty of computer science, Goce Delcev University,

Krste Misirkov No. 10-A Stip, Republic of North Macedonia

email: elena.gelova@ugd.edu.mk

Nikola Tuneski

Faculty of Mechanical Engineering,, Ss. Cyril and Methodius University in Skopje,

Karpoš II b.b., 1000 Skopje, Republic of North Macedonia email: nikola.tuneski@mf.edu.mk 